\title{
VARIACIÓN DE LAS VOCALES MEDIAS EN EL ESPAÑOL HABLADO EN LA CIUDAD DE LA HABANA EVIDENCIA FONÉTICO-ACÚSTICA
}

El vocalismo de las hablas del Caribe hispánico ha sido descrito de diversas maneras en los estudios fonológicos. En el caso del español de Cuba, nos interesan esencialmente dos fenómenos: la apertura de vocales ante aspiración y/o elisión de /s/ en final de sílaba, y el posible alargamiento vocálico cuando se da la elisión de /s/. Respecto de la apertura, Tomás Navarro Tomás reconoció, para el español de Puerto Rico, que "en posición final ante pausa, la $s$ puertorriqueña desaparece normalmente en el habla popular sin dejar otra huella que la de la abertura de la vocal con la que la palabra termina"1. Respecto de esta apreciación, María Vaquero señala que Navarro Tomás "oyó en el Caribe los timbres vocálicos del andaluz oriental, interpretados por él mismo, como indicios de reajuste morfofonológico en el sur de España y propuso para la isla antillana la misma interpretación" " Navarro Tomás había planteado un desdoblamiento vocálico para el andaluz consistente en la creación de una oposición entre vocales cerradas y abiertas, en la que estas últimas cifraban "la función semántica de la consonante desaparecida"3 (es decir, la /s/). Así, las oposiciones morfológicas singular/plural (tiempo/ tiempos) y segunda/tercera persona verbales (comes/come) estarían dadas por la distinción fónica de timbre cerrado/abierto de la vocal en posición final de palabra. Sin embargo, otros autores no reconocen el desdoblamiento vocálico en las hablas del Caribe.

1 El español en Puerto Rico, Universidad de Puerto Rico, Río Piedras, 1948, p. 73 .

2 En "Antillas", en Manual de dialectología hispánica. El español de América, ed. M. Alvar, Ariel, Barcelona, 1996, pp. 51-67.

3 "Desdoblamiento de fonemas vocálicos", Revista de Filología Hispánica, 1 (1939), 165-167. 
Vaquero, por ejemplo, advierte que "ni Henríquez Ureña ni Jiménez Sabater hacen referencia al desdoblamiento fonológico en el español de República Dominicana"4 . Asimismo, Cristina Isbasescu en su fonología descriptiva del español de Cuba no menciona ese desdoblamiento, aunque sí señala que la vocal final puede cambiar su timbre cuando hay elisión de $/ \mathrm{s} / 5$.

Respecto del alargamiento de vocales, es Robert Hammond quien plantea explícitamente una hipótesis de alargamiento vocálico compensatorio (en adelante AVC) como resultado de la elisión de /s/ para el español cubano ${ }^{6}$. Esta hipótesis se retoma en diversos trabajos dedicados a la descripción de hablas caribeñas ${ }^{7}$ para describir nuevos datos y argumentarlos con base en distintos enfoques teóricos. Desde esta perspectiva, en el marco de la fonología $S P E^{8}$ el proceso de alargamiento quedaría formalizado por la siguiente regla que, según Hammond, tendría un carácter global ${ }^{9}$ :

(1) $\mathrm{V} \rightarrow \mathrm{V}: / \ldots \mathrm{C}$

Esta regla tendría como condición sine qua non la aplicación previa de una regla de elisión de /s/:

$$
/ \mathrm{s} / \rightarrow \varnothing /[] \sigma
$$

La anterior sería, en términos de Hammond, una "condición global acompañante”, de la regla (1); esta última tendría el

4 M. VAQuero, op. cit., pp. 55-56.

5 El español de Cuba. Observaciones fonéticas y fonológicas, Sociedad de Lingüística Románica, Bucarest, 1968.

6 "En torno a una regla global en la fonología del español de Cuba", en Estudios sobre la fonología del español del Caribe, eds. R. Núñez Cedeño, J. Guitart e I. Páez Urdaneta, La Casa de Bello, Caracas, 1986, pp. 31-39, y "La fonología multidimensional y el alargamiento compensatorio", Revista Interamericana, 1988, núm. 19, 67-76.

7 Entre dichos trabajos están los de David Olmstead, "A note on the dialect of Regla, Cuba”, H, 37 (1954), 293-294; C. Isbasescu, op. cit., p. 46; Rafael del Rosario, El español de América, Troutman Press, Sharon, Connecticut, 1970, p. 83, y R. HAmmond, "An experimental varification on the phonemic status of open and closed vowels in Caribbean Spanish", en Corrientes actuales de la dialectología del Caribe hispánico, ed. H. López Morales, Editorial Universitaria, Río Piedras, 1978, pp. 117-119.

8 NoAm ChOmsky \& Morris Halle, The sound pattern of English, Harper \& Row, New York, 1968.

9 R. HAmmond, "En torno a una regla global", pp. 37-39. 
poder de "examinar" las derivaciones, de tal modo que sólo se aplicaría en aquellas en las que se hubiere aplicado la regla (2).

De esta manera, se tendrían derivaciones como las siguientes:

$\begin{array}{lcc} & \text { /peska/ } & \text { /kosta/ } \\ \text { Regla (2) } & \text { pe ka } & \text { ko ta } \\ \text { Regla (1) } & \text { pe:ka } & \text { ko:ta } \\ & \text { [pe:ka] } & {[\text { ko:ta] }}\end{array}$

Las reglas enunciadas en (1) y (2) predicen a priori un alargamiento vocálico generalizado a nivel de superficie, pero no dan cuenta de la variación que involucra la realización fonética del proceso en el español habanero. Desde una perspectiva no lineal, Rafael Núñez Cedeño ${ }^{10}$ intenta explicar el fenómeno por medio de la asociación de la posición prosódica $\mathrm{X}$ vacía, dejada por la /s/, a la vocal precedente:

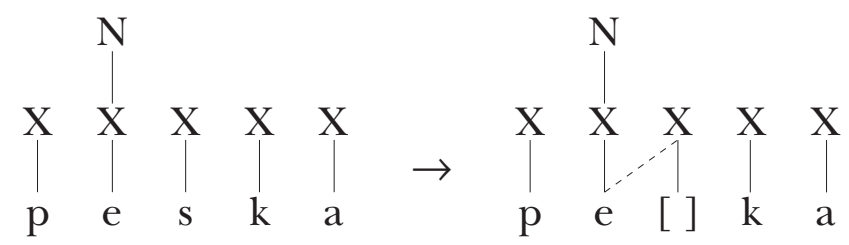

FigurA 1. Alargamiento vocálico compensatorio.

$\mathrm{Al}$ asociarse la $\mathrm{X}$ vacía a los rasgos de la /e/, esta última se convertiría, naturalmente, en una vocal larga, pues quedaría asociada a dos unidades temporales. Sin embargo, este planteamiento tampoco da cuenta de la complejidad del fenómeno porque no hace explícita la relación que hay entre el contexto que favorece la elisión de /s/ y el efecto de este proceso en la calidad de la vocal precedente. Con estos hechos como punto de partida, presentamos en este artículo los resultados de un estudio piloto sobre la variación de las vocales medias /e/ y /o/ del español hablado en la ciudad de La Habana. Los objetivos son dos: 1) proporcionar evidencia acústica y estadística que confirme

10 "Alargamiento vocálico compensatorio en el español cubano: un análisis autosegmental”, en Studies in Caribbean Spanish dialectology, eds. R. Hammond \& R. Resnick, Georgetown University Press, Washington, DC, 1988, pp. 97-102; y R. Núñez Cedeño y Alfonso Morales Front, Fonología generativa contemporánea de la lengua española, Georgetown University Press, Washington, DC, 1999, pp. 64-67. 
la ocurrencia o no de alargamiento vocálico compensatorio y de apertura vocálica como fenómenos de variación fónica en el habla de la ciudad de La Habana y 2) hacer notar que un sistema de reglas (o de representaciones) per se no explica la variación de las vocales medias /e/ y /o/ resultado de la elisión de /s/. Proponemos que la explicación se puede encaminar desde un enfoque multidimensional de la fonología, siempre y cuando se aclare la variación fonética de las vocales en contexto de /s/ aspirable de tal modo que sea posible afirmar, en lo que respecta a la ocurrencia del AVC, una hipótesis de peso silábico para el español habanero ${ }^{11}$. En efecto, nuestra propuesta es que el AVC pudiera ser un fenómeno que en el español hablado en la ciudad de La Habana sería una manifestación del peso silábico si asumimos que el segmento /s/ en posición de coda cuenta como mora, de tal suerte que al elidirse /s/, la mora que queda libre se asocia -como mecanismo compensatorio- a la vocal precedente y por eso se alarga. Lo anterior significaría que la elisión de /s/ no afecta la estructura prosódica del sistema en cuestión porque se compensa en el nivel moraico. En este sentido, la variante habanera parecería mostrar que el sistema compensa en la estructura prosódica las pérdidas segmentales, en la medida en que se comporta como un sistema de peso múltiple que tiene que rastrearse en distintos estratos de su fonología; es decir, en el esqueleto prosódico, en la melodía segmental y en la jerarquía de rasgos.

Como señalamos al principio, un fenómeno comúnmente asociado a la aspiración y elisión de /s/ en final de sílaba es el cambio de timbre de la vocal precedente, más concretamente, la apertura de dicho segmento. Esta es una afirmación frecuente en los trabajos sobre aspiración y elisión de /s/ en hablas hispánicas, pero no siempre se encuentra al respecto evidencia de carácter instrumental. En el caso del andaluz, por ejemplo, los trabajos de Martínez Melgar y de Herrera y Galeote ${ }^{12}$ muestran que, en efecto, se verifica una apertura que se manifiesta acústicamente por un aumento en la frecuencia del pri-

11 Elizabeth Santana Cepero, Fonología y peso silábico en el español hablado en Ciudad de La Habana, tesis doctoral en proceso, El Colegio de México.

12 A. Martínez Melgar, "Estudio experimental sobre un muestreo del vocalismo andaluz", EFE, 2 (1986), 194-248, y "El vocalismo del andaluz oriental”, EFE, 6 (1994), 13-64; y Esther Herrera Zendejas y Manuel Galeote, "Estudio instrumental del vocalismo en la Andalucía Oriental", AnMal, 26 (2003), 379-398. 
mer formante (F1); las vocales que muestran un aumento más pronunciado del F1 son, precisamente, las que abordamos en el presente estudio, /e/ y /o/, y son las que suelen presentar una mayor variación en cuanto a su realización fonética. Por el contrario, el aumento en el F1 es menos acusado en el caso de /i/ y de $/ \mathrm{u} /$, e inexistente en el caso de /a/, la cual al no poder ser más abierta tiende a centralizarse ${ }^{13}$.

\section{Metodología y CORPUS}

De un total de 1200 casos de contextos de /s/ aspirable y /s/ no aspirable registrados y transcritos en un corpus de habla espontánea del español de la ciudad de La Habana, seleccionamos una muestra de 80 , los cuales se encuentran en las 50 oraciones que aparecen en el Apéndice de este trabajo. Estas oraciones fueron elicitadas posteriormente con una hablante nativa de la variante habanera que no fue entrevistada en el corpus original, de 31 años de edad, residente en La Habana toda su vida, que estaba de visita en México por pocos días. Las oraciones se le presentaron mezcladas aleatoriamente para su lectura; se grabaron tres repeticiones de cada oración directamente en el CSL de Kay, con la señal digitalizada a $11000 \mathrm{~Hz}$. Para ubicar los fenómenos de alargamiento y apertura vocálicos en el material recopilado consideramos los siguientes contextos ${ }^{14}$ :

(4) a. É y Ó (tónicas) con /s/ aspirable como en pesca y costa.

b. E y $\mathbf{O}$ (átonas) con $/ \mathrm{s} /$ aspirable como en escuelas y otros pescados.

c. é y ó (tónicas) sin /s/ aspirable como peca y fotos.

$d$. e y o (átonas) $\sin / \mathrm{s} /$ aspirable como en dice buque y conocimientos.

Los índices acústicos que tomamos en cuenta para describir fonéticamente la variación de /e/ y /o/ en estos contextos fue-

13 Véanse los resultados de E. Herrera y M. Galeote, op. cit., p. 391.

14 Los símbolos É, Ó, E, O, é, ó, e, o, son arbitrarios y representan únicamente variantes vocálicas seguidas de /s/ aspirable, en el caso de las mayúsculas, y no seguidas de /s/ aspirable en el caso de las minúsculas. Es decir, no representan alófonos de las vocales medias. Así pues, no nos comprometemos con el estatus de estos segmentos dentro del sistema, lo cual sería objeto de otro trabajo. 
ron la duración de la vocal y la altura de los primeros tres formantes (F1, F2 y F3), medidos a partir del análisis LPG (Linear Prediction Coefficient o Coeficiente de Predicción Lineal) para determinar su grado de apertura y su anterioridad-posterioridad. Con los resultados obtenidos se realizó estadística descriptiva y análisis de varianza (ANOVA).

\section{Resultados}

Comentaremos los resultados del análisis acústico y estadístico a la luz de los fenómenos de alargamiento y de apertura. En este sentido, la evidencia acústica y la estadística permitieron obtener un diagnóstico cercano a lo que ocurre con la variación de las vocales /e/ y /o/ del dialecto habanero.

\section{Resultados relacionados con le/}

Respecto de la variación vocálica de /e/, especificada como anterior, media y no labializada, reconocimos cuatro formas fonéticas que corresponden a cuatro posibilidades de variación:

(5) a. É como la de $p[\hat{\mathbf{E}}] s c a, p[\hat{\mathbf{E}}] z$ pargo, vocal media, anterior, tónica, $x$ abierta, $y$ larga (Figura 2).

$b$. é como la de $p[\mathbf{e}] c a, p[$ é] $c e s$, vocal media, anterior, tónica, $x$ abierta, $y$ larga (Figura 3).

c. $\mathbf{E}$ como la de $[\mathbf{E}]$ scuelas, amor $[\mathbf{E}]$ s perdidos, vocal media anterior, átona, $x$ abierta, $y$ larga (Figura 4).

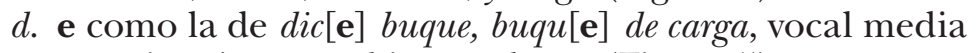
anterior, átona, $x$ abierta, $y$ larga (Figura 5).

Las $x$ y las $y$ en los parámetros de apertura y alargamiento indican variabilidad en el mayor, menor o cero grado de apertura y en la mayor o menor duración de la vocal. La duración media de cada una de las variantes señaladas fue la siguiente ${ }^{15}$ :

15 Donde $S^{2}=$ varianza y $S=$ desviación estándar. 
TABLA 1

Duración de las realizaciones de lel

\begin{tabular}{|c|c|c|c|}
\hline & Duración media & $S^{2}$ & $S$ \\
\hline $\mathbf{E}$ & $74.4 \mathrm{msg}$ & 52.2 & 7.2 \\
\hline $\mathbf{e}$ & $63.5 \mathrm{msg}$ & 187.7 & 13.7 \\
\hline $\mathbf{E}$ & $74.4 \mathrm{msg}$ & 522.3 & 22.8 \\
\hline $\mathbf{e}$ & $69.1 \mathrm{msg}$ & 538.3 & 23.2 \\
\hline
\end{tabular}

En cuanto al parámetro de longitud en las realizaciones correspondientes a /e/, la duración media daría apoyo a la hipótesis de peso silábico, al menos en parte. Ello, debido a que el alargamiento resultó significativo al menos para las vocales $\mathbf{E}$ tónica y $\mathbf{E}$ átona (en contexto de /s/ aspirable), las cuales resultaron en promedio ser más largas que las vocales é tónica y e átona (en contexto $\sin$ /s/ aspirable), lo que nos hablaría, en principio, de una relación significativa entre el contexto de /s/ aspirable y la duración. La diferencia entre las medias de duración de $\mathbf{E}$ y $\mathbf{E}$ fue nula, pero entre estas dos y la é, fue de $11 \mathrm{msg}$, y entre ambas y la e la diferencia fue de $4 \mathrm{msg}$. El cálculo de ANOVA indicó que la diferencia entre las medias de duración de É y é fue significativa, aunque la diferencia entre las medias de $\mathbf{E}$ y e no lo fue. Así, los resultados obtenidos le dan un apoyo sólo parcial a la hipótesis del alargamiento compensatorio, en vista de que el alargamiento de la /e/ en contexto de /s/ aspirable -si lo hay- sólo resulta significativo cuando la vocal es tónica ${ }^{16}$.

Consideremos ahora la abertura de las vocales; en las Figuras 2 a 5 observamos un ejemplo de cada una de las cuatro variantes de /e/ que hemos especificado en (5), y de las cuales hemos incluido el oscilograma, el espectrograma y el LPC. Cabe señalar que la medición de los F1, F2 y F3 se realizó con base en la parte más estable del formante (generalmente, el centro del mismo), de la cual se extrajo el LPG:

16 Hay que señalar que, pese a este resultado, la media que evidencia el alargamiento vocálico en esta variante no corresponde a los valores de duración en lenguas donde la longitud es fonológicamente contrastiva como, por ejemplo, el checo o el húngaro, en las que la realización de una vocal larga tiene una duración de aproximadamente una y media veces la de una vocal breve. Si tomamos como base este criterio, el alargamiento que ocurre en el español hablado en La Habana, registrado en contexto de /s/ aspirable en interior de palabra, no es claramente compensatorio, con lo cual queda en duda su pertinencia fonológica, como mostraremos en lo que sigue. 

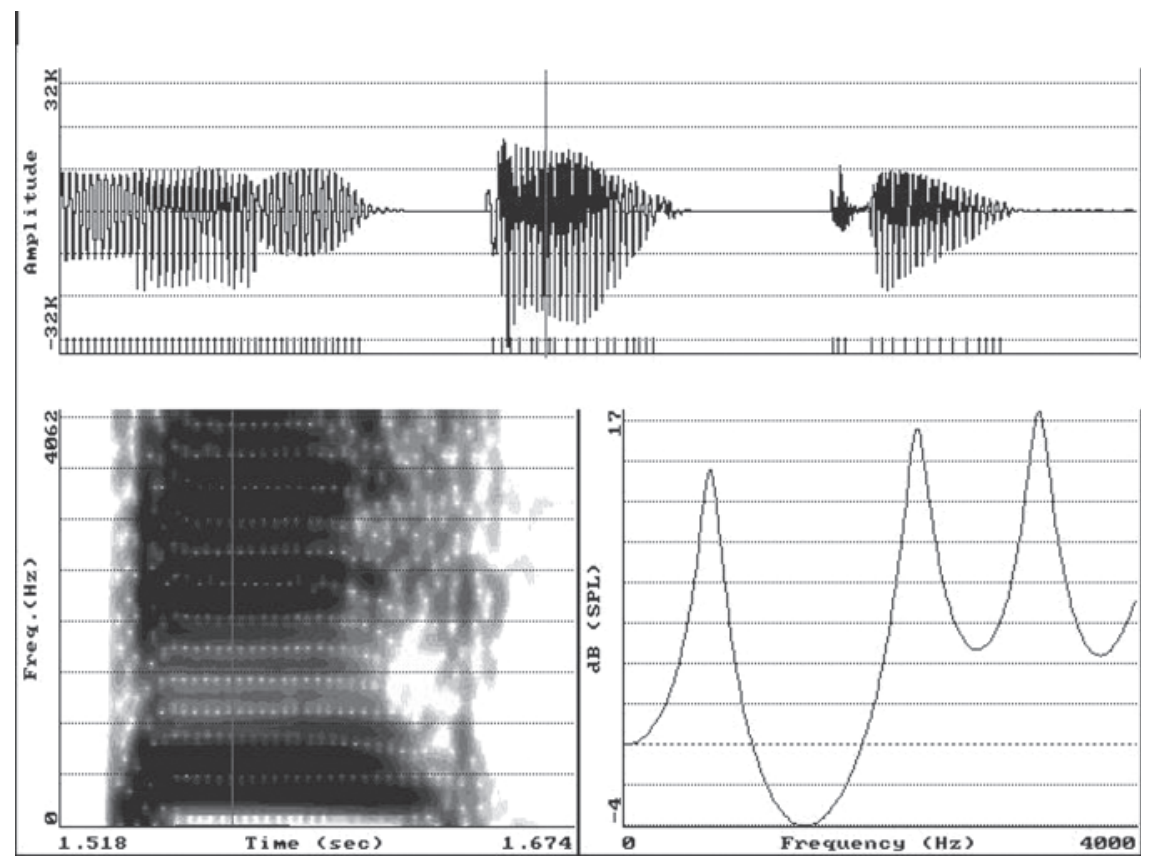

Figura 2. Vocal É en $p[\mathbf{E}] s c a$.
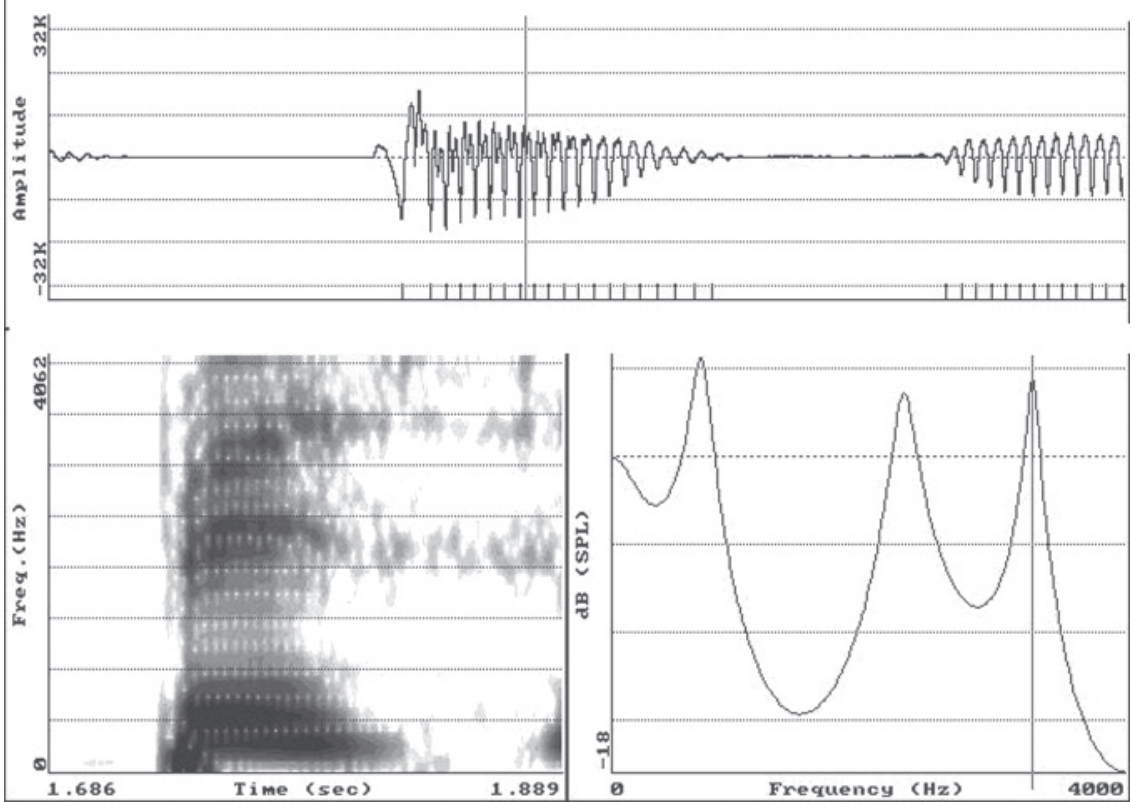

Figura 3. Vocal é en p[é]ces. 

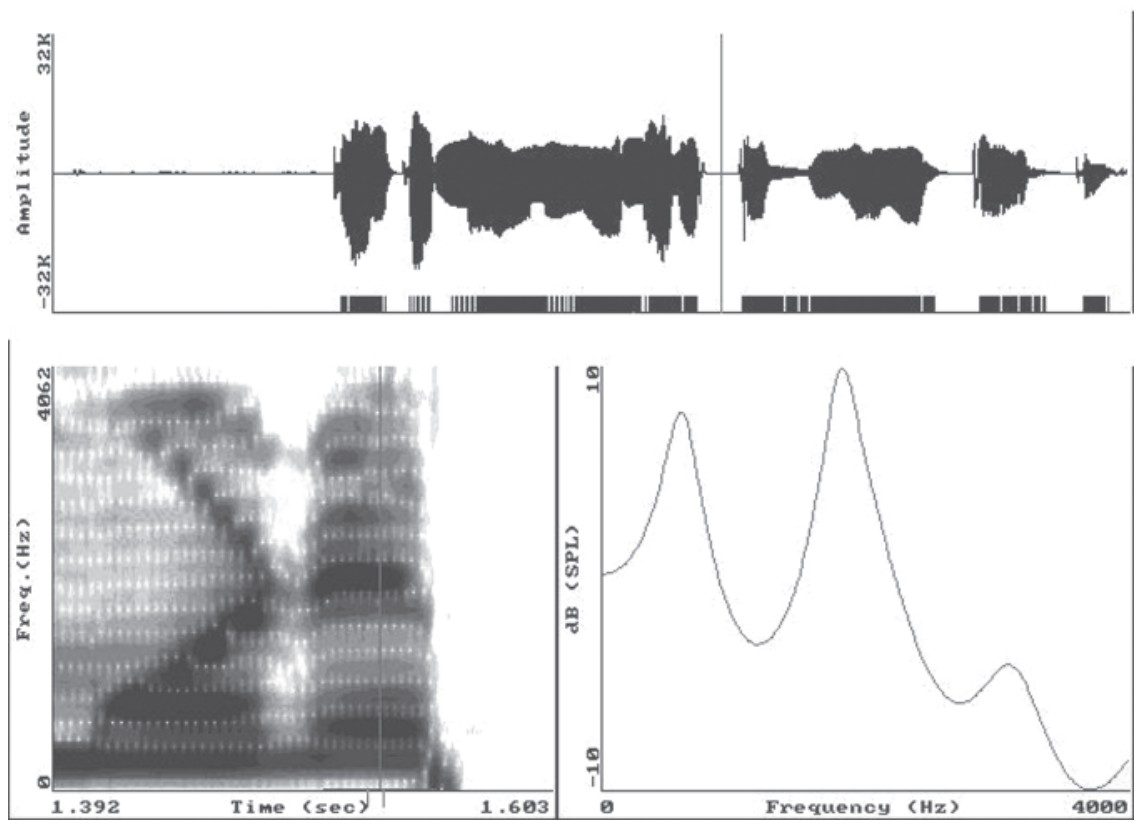

Figura 4. Vocal E en amor[E]s perdidos.

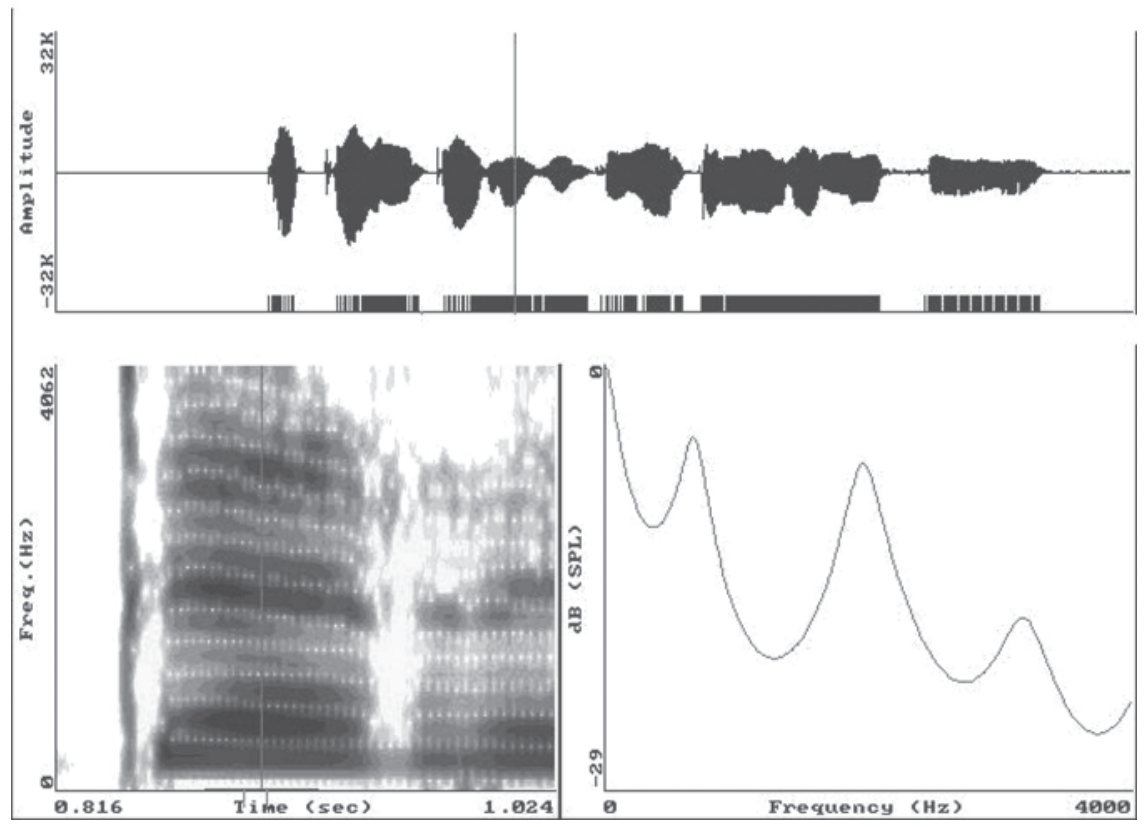

Figura 5. Vocal e en buqu[e] de carga. 
Los resultados de la medición de la frecuencia del primer y el segundo formantes fueron los siguientes:

TABLA 2

Estructura formántica de las realizaciones de le/

Frecuencia (media) de F1

\begin{tabular}{|c|c|c|c|}
\hline & & $S^{2}$ & $S$ \\
\hline $\mathbf{E}$ & $650.9 \mathrm{~Hz}$ & 15117.6 & 38.9 \\
\hline é & $694.7 \mathrm{~Hz}$ & 5622.4 & 74.9 \\
\hline
\end{tabular}

Frecuencia (media) de F1

\begin{tabular}{|c|c|c|c|}
\hline & & $S^{2}$ & $S$ \\
\hline $\mathbf{E}$ & $645.2 \mathrm{~Hz}$ & 19154.1 & 138.3 \\
\hline $\mathbf{e}$ & $668.0 \mathrm{~Hz}$ & 2627.3 & 51.2 \\
\hline
\end{tabular}

Frecuencia (media) de F2

\begin{tabular}{|c|c|c|c|}
\hline & & $S^{2}$ & $S$ \\
\hline $\mathbf{E}$ & $2192.2 \mathrm{~Hz}$ & 30580.1 & 174.8 \\
\hline $\mathbf{e}$ & $2254.6 \mathrm{~Hz}$ & 26092.0 & 161.5 \\
\hline
\end{tabular}

Frecuencia (media) de F2

\begin{tabular}{|c|c|c|c|}
\hline & & $S^{2}$ & $S$ \\
\hline $\mathbf{E}$ & $2104.2 \mathrm{~Hz}$ & 21607.2 & 146.9 \\
\hline $\mathbf{e}$ & $2040.7 \mathrm{~Hz}$ & 21384.4 & 146.2 \\
\hline
\end{tabular}

En cuanto a la apertura, las diferencias en la altura media del F1 entre las realizaciones de /e/ en contextos de /s/ aspirable $(\hat{\mathrm{E}}, \mathrm{E})$ versus las realizaciones de la misma vocal en contextos $\sin / \mathrm{s} /$ aspirable (é, e) no resultaron significativas, según indicó el cálculo de ANOVA, por lo que dicho contexto no parece ejercer mucha influencia en la apertura de la /e/. Incluso vemos que las realizaciones $\sin / \mathrm{s} /$ aspirable (é, e) aparecieron en promedio más abiertas que É y E, contrario a lo que se podría suponer ${ }^{17}$. Respecto de los resultados obtenidos con la frecuencia del

17 Diversos trabajos han descrito la relación entre la aspiración de /s/ y el timbre vocálico. El planteamiento común en dichos trabajos es que hay una relación directa entre la aspiración y la apertura vocálica. Sin embargo, como se observa en los datos de (7), esa relación no se cumple en el mate- 
F2, la diferencia entre las realizaciones de /e/ en contexto de /s/ aspirable y $\sin / \mathrm{s} /$ aspirable no resultaron significativas, de ahí que tampoco el contexto de /s/ aspirable parece ejercer alguna influencia en la anterioridad/posterioridad de las realizaciones de /e/.

Es por los hechos anteriores que reconocimos cuatro variantes de /e/ que combinan el rasgo de abertura y la prominencia acentual; sólo una de esas variantes -la $\mathbf{E}-$, resultó significativamente alargada (véase Figura 2 y los datos de la Tabla 2). La intensidad en relación con la frecuencia muestra que la apertura no parece ser sensible al acento, sino más bien al contexto de /s/ aspirable: en sílaba prominente, é tónica se abre más que É, y en sílabas átonas es e la que se abre más que $\mathbf{E}$. Visto en conjunto, podemos afirmar que la variación en la apertura de la serie de vocales medias anteriores tiene su punto máximo en la é tónica más abierta (con valores de frecuencia más cercanos a una /a/, por ejemplo), y su punto mínimo en la $\mathbf{E}$ átona menos abierta (independientemente de que sea una átona alargada). No obstante, es necesario señalar que el análisis ANOVA indicó que las diferencias entre las medias del F1 de É y é, E y e no resultaron significativas. Respecto de los datos para el segundo formante (F2), la conclusión es similar: el contexto de /s/ aspirable y la tonicidad no parecen ejercer un efecto claro en la anterioridad o posterioridad de las realizaciones de /e/.

rial que hemos analizado de la variante habanera; véanse JoHn J. OHALA, "Phonetic explanation for nasal sound patterns", en Nasalfest. Papers from a Symposium on nasals and nasalization, eds. C.M. Ferguson, L.M. Hymany \& J.J. Ohala, Stanford University Press, Palo Alto, CA, 1975, pp. 289-316; VictoriA Marrero, "Estudio acústico de la aspiración en español", RFE, 70 (1990), 345-397; Antonio Quilis, Tratado de fonología y fonética españolas, Gredos, Madrid, 1993; Yolanda Rodríguez Cadena, "El efecto de la aspiración sobre la tonía intrínseca de las vocales en el español del Caribe", en La tonía: dimensiones fonéticas y fonológicas, eds. E. Herrera y P. Martín Butragueño, El Colegio de México, México, 2003, pp. 75-94. Por otra parte, es también un lugar común en la literatura sobre el vocalismo español la suposición de que las vocales se articulan más abiertas en sílabas cerradas. Sin embargo, para algunas variantes se ha mostrado que esto no es necesariamente cierto; al respecto, véase, por ejemplo, E.A. MAdrid Servín y Mario A. MARín RodríGUEz, "La estructura formántica de las vocales del español de la ciudad de México", en Temas de fonética instrumental, ed. E. Herrera Zendejas, El Colegio de México, México, 2001, pp. 39-58. 
Resultados relacionados con /o/

En la variación de /o/, especificada como posterior, media y labializada, constatamos cuatro realizaciones:

(6) a. Ó como la de $c[\mathbf{O}] s t a, p[\mathbf{O}]$ stes, vocal media, posterior, tónica, $x$ abierta, $y$ larga (Figura 6).

$b$. ó como la de $f$ [ó]tos, $y$ [óo] soy, vocal media, posterior, tónica, $x$ abierta, $y$ larga (Figura 7 ).

c. $\mathbf{O}$ como la de $\operatorname{larg}[\mathbf{O}]$ s, otr $[\mathbf{O}]$ s pescados, vocal media, posterior, átona, $x$ abierta, $y$ larga (Figura 8).

$d$. o como la de $c[\mathbf{o}]$ nocimiento, osteop $[\mathbf{o}]$ rósis, vocal media, posterior, átona, $x$ abierta, $y$ larga (Figura 9).

Respecto del parámetro de longitud, la media de la duración inclina la balanza hacia el rechazo de la hipótesis del alargamiento compensatorio en la serie de realizaciones de /o/:

TABLA 3

Duración de las realizaciones de /o/

\begin{tabular}{|c|c|c|c|}
\hline & Duración media & $S^{2}$ & $S$ \\
\hline Ó & $67.1 \mathrm{msg}$ & 273.6 & 16.5 \\
\hline ó & $69.3 \mathrm{msg}$ & 127.0 & 11.2 \\
\hline $\mathbf{O}$ & $55.9 \mathrm{msg}$ & 111.0 & 10.5 \\
\hline $\mathbf{o}$ & $58.2 \mathrm{msg}$ & 175.1 & 13.2 \\
\hline
\end{tabular}

Salta a la vista que las realizaciones de /o/ en contextos con /s/ aspirable (Ó, O) no sólo no se alargaron, sino que incluso resultaron, en promedio, más breves que sus contrapartes en contextos sin /s/ aspirable (ó, o). Por supuesto, las diferencias entre las realizaciones en ambos contextos no fueron significativas. En cuanto a la medición de las frecuencias de los dos primeros formantes, los resultados fueron los siguientes: 
TABla 4

Estructura formántica de las realizaciones de /o/

Frecuencia (media) de F1

\begin{tabular}{|c|c|c|c|}
\hline & & $S^{2}$ & $S$ \\
\hline Ó & $660.0 \mathrm{~Hz}$ & 2948.8 & 54.3 \\
\hline $\mathbf{o}$ & $667.8 \mathrm{~Hz}$ & 8212.4 & 90.6 \\
\hline
\end{tabular}

Frecuencia (media) de F1

\begin{tabular}{|c|c|c|c|}
\hline & & $S^{2}$ & $S$ \\
\hline $\mathbf{O}$ & $623.7 \mathrm{~Hz}$ & 9026.2 & 95.0 \\
\hline $\mathbf{0}$ & $592.8 \mathrm{~Hz}$ & 5399.0 & 73.4 \\
\hline
\end{tabular}

Frecuencia (media) de F2

\begin{tabular}{|c|c|c|c|}
\hline & & $S^{2}$ & $S$ \\
\hline Ó & $1259.7 \mathrm{~Hz}$ & 10421.5 & 102.0 \\
\hline ó & $1444.3 \mathrm{~Hz}$ & 53392.6 & 231.0 \\
\hline
\end{tabular}

Frecuencia (media) de F2

\begin{tabular}{|c|c|c|c|}
\hline & & $S^{2}$ & $S$ \\
\hline $\mathbf{O}$ & $1445.5 \mathrm{~Hz}$ & 41176.7 & 202.9 \\
\hline $\mathbf{0}$ & $1454.0 \mathrm{~Hz}$ & 84333.7 & 290.4 \\
\hline
\end{tabular}

Observamos que la apertura es un fenómeno variable para las realizaciones de /o/, mientras que el alargamiento no lo es. Las Figuras 6, 7, 8 y 9 muestran ejemplos del análisis acústico de las variantes especificadas en (8): 

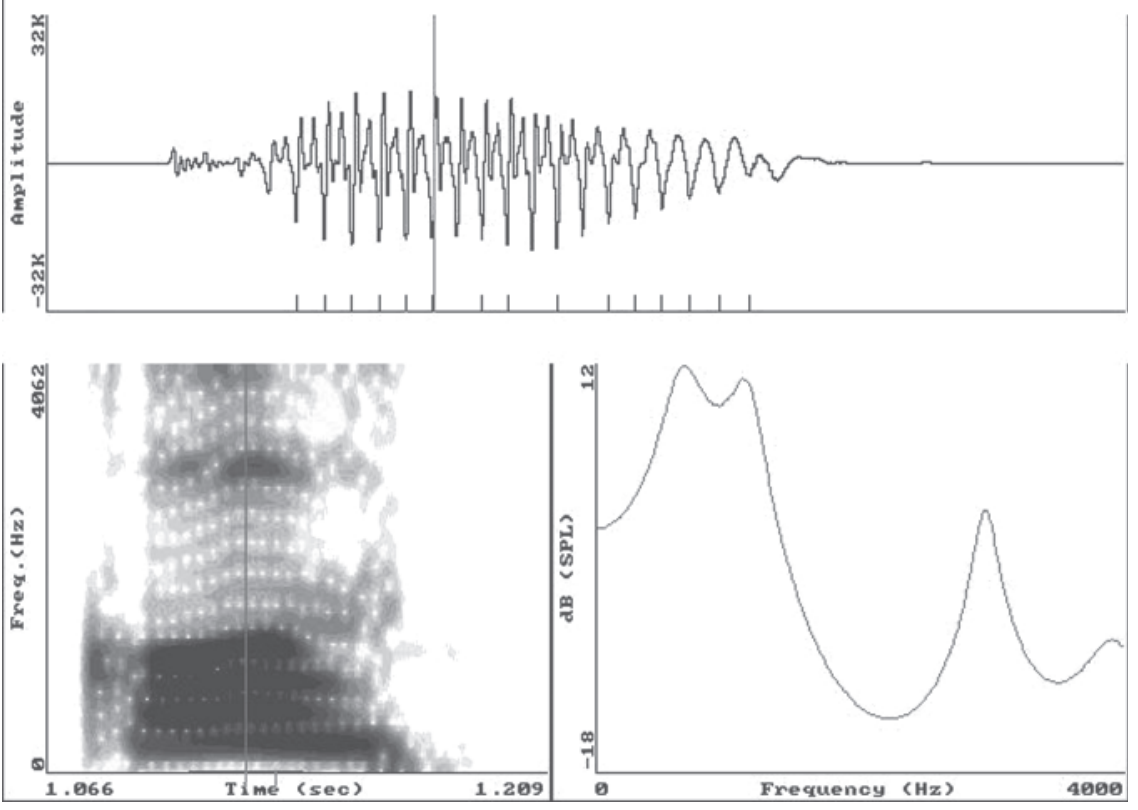

Figura 6. Vocal Ó en $c[\mathbf{O}] s t a$.
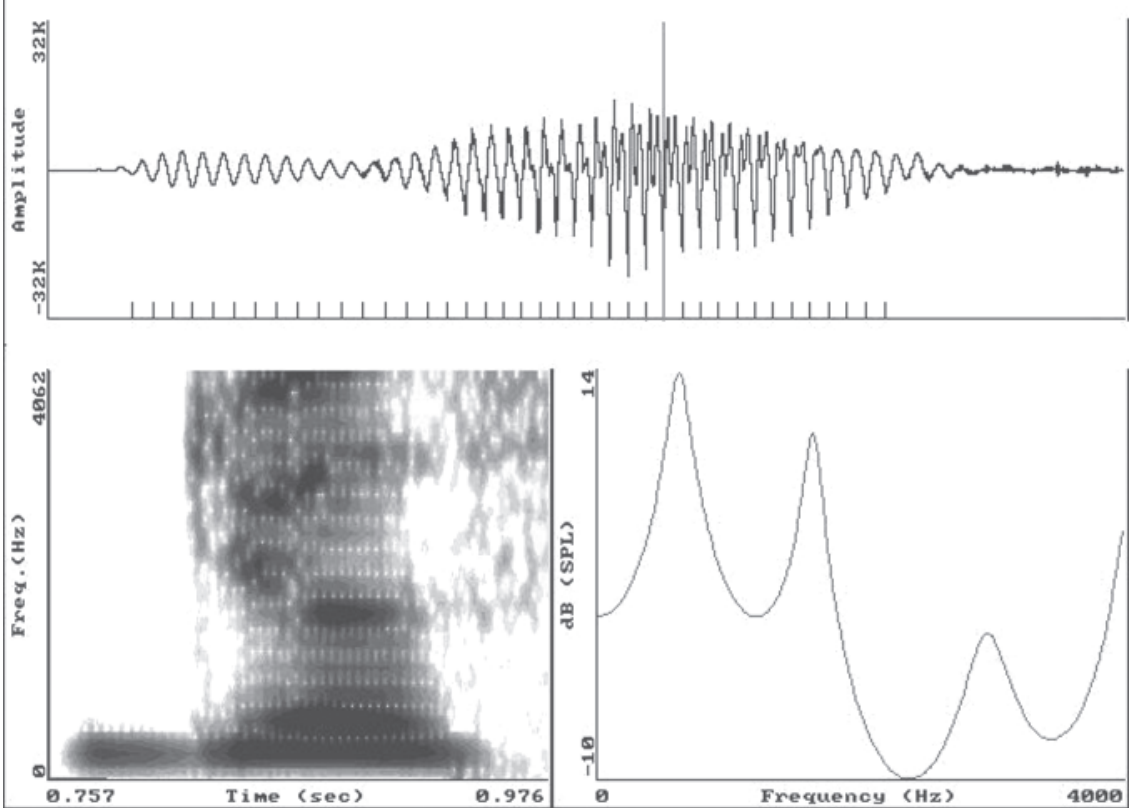

Figura 7. Vocal ó en $y[\mathbf{o}]$ soy. 

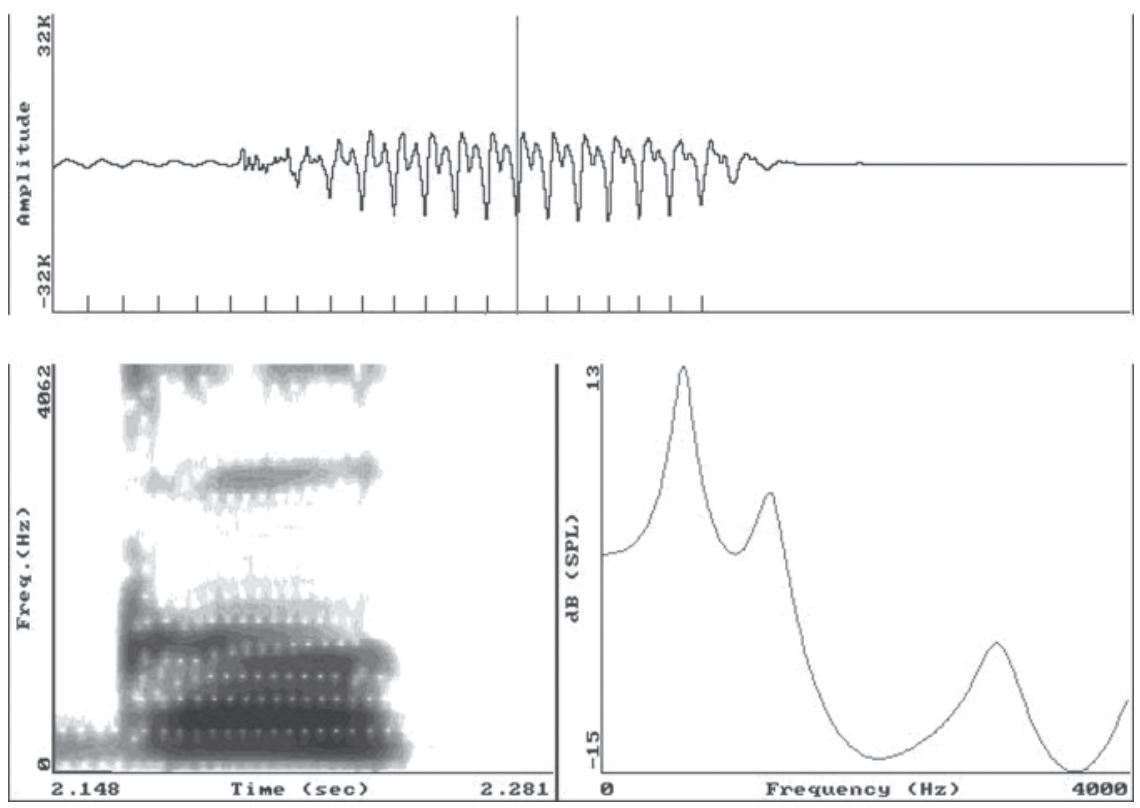

Figura 8. Vocal $\mathbf{O}$ en $\operatorname{larg}[\mathbf{O}] s$.

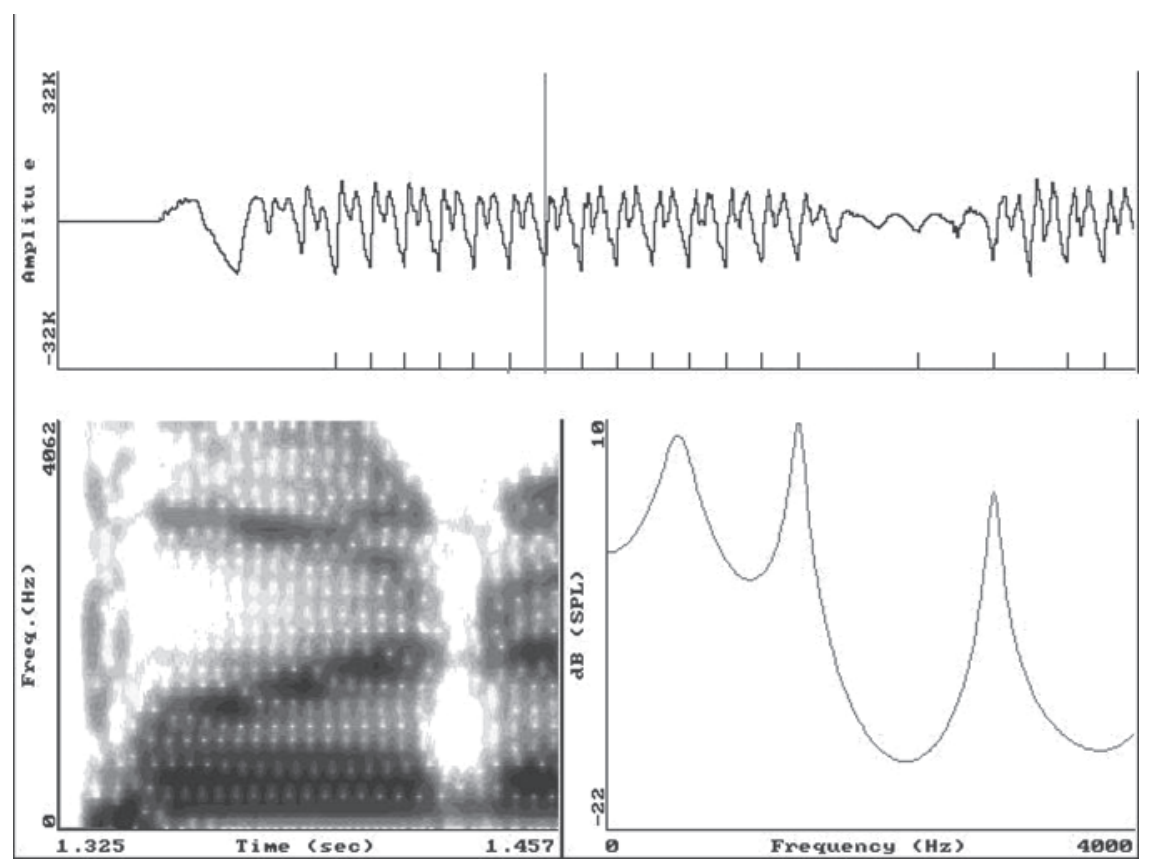

Figura 9. Vocal o en osteop[o]rosis. 
Los resultados obtenidos de la medición de la altura de los formantes nos permite corroborar que en la variación de las vocales medias del español habanero hay una asimetría en cuanto a la apertura, puesto que, mientras la vocal media anterior muestra realizaciones un poco más abiertas (si bien estadísticamente no significativas) en el contexto sin /s/ aspirable (e, é), las realizaciones de la vocal posterior (o, ó) en el mismo contexto no muestran tendencia a la abertura; por el contrario, es la realización en contexto de /s/ aspirable, la que muestra cierta abertura (la cual, sin embargo, tampoco resultó significativa).

Por lo que respecta a la información del segundo formante (F2), observamos que las realizaciones de /o/ tónica fuera del contexto de /s/ aspirable son notoriamente más anteriores que sus contrapartes en dicho contexto; de hecho, el cálculo ANOVA mostró que la diferencia entre los valores medios del F2 sí resultaron significativos, cosa que no ocurrió, como vimos, con las realizaciones de /e/ en el mismo contexto, lo cual crea otra asimetría en el comportamiento de las realizaciones de ambas vocales. Finalmente, las realizaciones átonas de /o/ no mostraron mayor diferencia en cuanto a la anterio$\mathrm{ridad} / \mathrm{posterioridad}$.

$\mathrm{Al}$ considerar en conjunto las realizaciones de las vocales medias en el material analizado lo que obtenemos es un panorama en el que predomina la dispersión. En la Figura 9 se muestran los valores para el F1 y la F2' de cada uno de los segmentos medidos ${ }^{18}$. Si bien es posible separar con claridad las realizaciones de /e/ y de /o/, vemos que no lo es tanto delimitar nítidamente zonas de dispersión para cada una de las realizaciones en los contextos con /s/ aspirable y sin ésta: se entremezclan sin que parezca que dicho contexto ejerce alguna influencia decisiva.

18 La F2' es el valor del F2 tomando en cuenta la influencia que sobre éste ejerce el F3. 


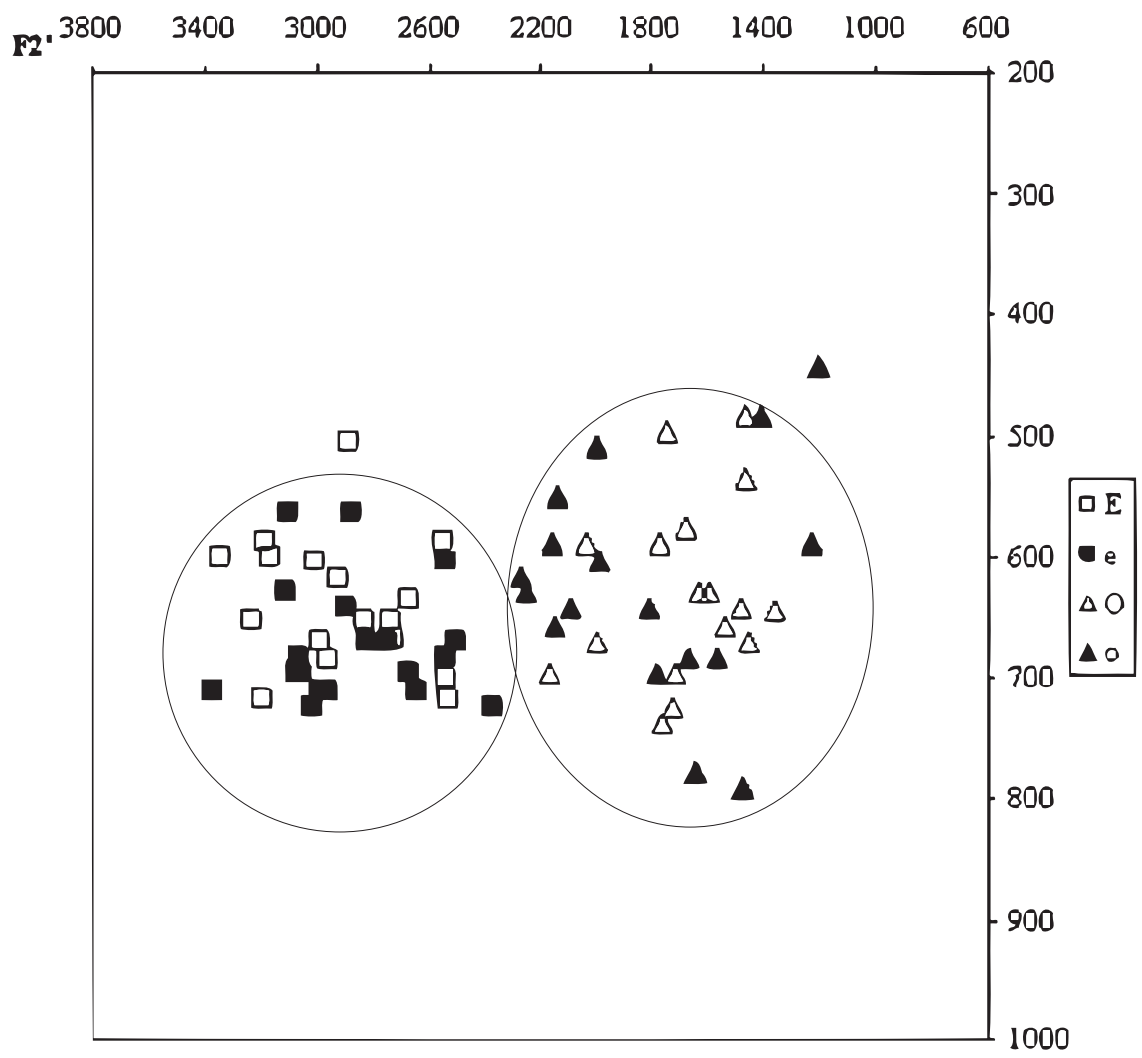

R1

Figura 10. Variación en las realizaciones de /e/ y de /o/.

Estos hechos de notoria variación fónica permiten, sin embargo, hacer algunas generalizaciones. En el siguiente apartado expondremos un posible camino que, desde la fonología teórica, pudiera explicar fenómenos y procesos de variación fónica como el alargamiento y la apertura vocálicos presentes en la variante del español caribeño estudiada aquí.

\section{AGERCAMIENTO A UNA EXPLICACión}

DESDE LA TEORÍA FONOLÓGICA

Con base en los resultados obtenidos en el presente estudio podemos asumir que no se puede explicar satisfactoriamente lo que sucede con la variación de las vocales medias /e/ y /o/ del español de La Habana (y, probablemente, del Caribe en general) formulando una única regla (hipotéticamente) global 
como la de (1) o estableciendo una representación como la de la Figura 1 que, a priori, confirman una hipótesis de alargamiento compensatorio. Esto es lo que la teoría fonológica ha hecho al citar ejemplos extraídos de trabajos acerca de distintas hablas caribeñas. Es cierto que dichos ejemplos -repetidos una y otra vez en la bibliografía sobre el tema- pueden sugerir, desde determinados enfoques teóricos, un comportamiento sistemático de las vocales medias del español habanero ${ }^{19}$. Sin embargo, con los resultados de este estudio piloto, lo que observamos es que las formas fónicas de las vocales involucradas en el fenómeno de aspiración de /s/ parecen tener un comportamiento que no comprueba del todo las predicciones hechas hasta el momento por la teoría fonológica. El alargamiento no se verifica fonéticamente siempre que se espera de acuerdo con el contexto, como tampoco se da la apertura concomitante a la elisión de la /s/ en todos los casos. Así lo muestran los datos graficados en la Figura 10. Estamos, entonces, ante un proceso de variación más complejo que requiere explicaciones que van más allá de una regla categórica sólo en apariencia.

Un modelo teórico que intente explicar casos de variación lingüística como los tratados en este trabajo, tiene que considerar "varios niveles de análisis, segmentales, y prosódicos, modelos multidimensionales que no pretendan reducir a un solo plano todos los fenómenos" 20 . Las propuestas teóricas revisadas que intentan explicar la variación de vocales medias en contexto de /s/ aspirable no parecen tomar en cuenta del todo hechos como los presentados aquí.

El alargamiento compensatorio expresado tanto por la regla (1) como por la representación de la Figura 1 puede, por supuesto, ser discutido desde un modelo multidimensional, particularmente bajo la forma de una hipótesis de peso silábico; para la variación de las vocales medias /e/ y /o/ del español habanero necesariamente tendremos que considerar estratos prosódicos, el nivel silábico y moraico, el estrato segmental y la jerarquía de rasgos $^{21}$. Por el momento sólo queremos resaltar el hecho de que

19 Los ejemplos frecuentemente citados son pescado/pecado; busque/ buque, pastilla/patilla. Véanse, por ejemplo, R. HAMmOND, En torno a una regla global..., pp. 33-34 y R. Núñez Cedeño y A. Morales Front, op. cit., p. 65.

20 P. Martín Butragueño, Variación lingüistica y teoría fonológica, El Colegio de México, México, 2002, pp. 181-182.

21 Esta explicación multidimensional es tema de una investigación más amplia expuesta en E. Santana Cepero, op. cit. 
hay evidencias en el español habanero de que el alargamiento vocálico es más variable que compensatorio. Al tomar en cuenta lo anterior, la hipótesis de peso silábico basada en la teoría moraica ${ }^{22}$, por ejemplo, haría la predicción siguiente: una vez elidida la /s/ en palabras como pesca y costa, las respectivas vocales /e/ y /o/ se alargarían debido a que se asociaría a ellas la mora correspondiente a la /s/ en coda elidida; el proceso puede representarse así:
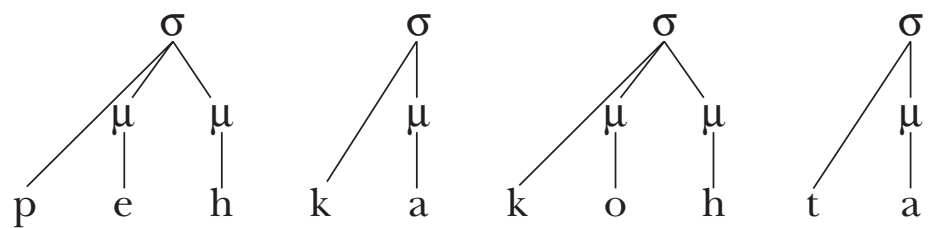

Figura 11. Estructura moraica y aspiración.
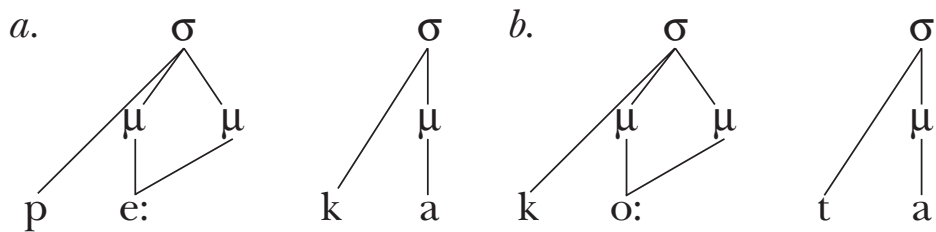

FIGURA 12. Estructura moraica y alargamiento.

Este proceso sería evidencia de lo que John McCarthy y Alan Prince denominan Condición de la Satisfacción Templática (Template Satisfaction Condition) ${ }^{23}$, el cual exigiría que la mora que queda sin ningún segmento asociado después de la elisión de /s/ se una a otro elemento de la melodía segmental, en lugar de perderse ${ }^{24}$. Sin embargo, lo que encontramos es un comportamiento variable del parámetro de longitud: en el caso de la /e/ hay un cierto alargamiento que no es sistemáticamente compensatorio; en el caso de la /o/, sencillamente no lo hay. Tam-

22 Bruce Hayes, "Compensatory lengthening in moraic phonology", LI, 20 (1989), 253-306.

23 "Prosodic morphology", en The handbook of phonological theory, ed. J.A. Goldsmith, Basil Blackwell, Oxford, 1995, pp. 318-366.

24 La Condición de Satisfacción Templática es uno de los tres principios de la Morfología Prosódica (Prosodic Morphology) planteados por J. McCarthy y A. Prince (art. cit.). 
poco observamos la ocurrencia de una apertura sistemática de las vocales en el contexto de /s/ aspirable (excepto en el caso de la $\mathbf{O}$ átona). En realidad, la ocurrencia de variantes más o menos alargadas y variantes más o menos abiertas son muestra de la gran dispersión que presentan las vocales medias en el sistema fónico del español habanero, en el cual podemos reconocer zonas muy inestables que marcan la variación misma y zonas más estables donde aparecen las variantes más discretas, aquellas que nos permiten seguir reconociendo a /e/ como anterior y a /o/ como posterior. Es decir, pese a la variación dispersa no hay desdoblamiento vocálico en este sistema. La formalización fonológica del proceso desencadenado por la aspiración podría plantearse así:

$a$.

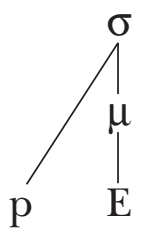

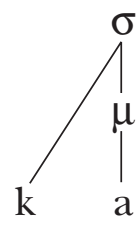

$b$.
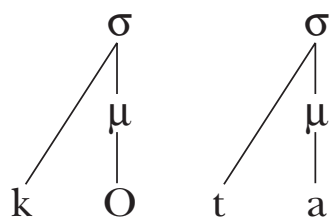

Figura 13. Estructura moraica de las realizaciones $\mathrm{de} / \mathrm{e} / \mathrm{y} / \mathrm{o} / \mathrm{en}$ contexto de /s/ aspirable.

Lo anterior, si tomamos en cuenta que el alargamiento de /e/ no fue estadísticamente significativo en todos los casos y que las realizaciones de /o/ no reflejan fonéticamente este mismo proceso, explicaría por qué el alargamiento vocálico no es en realidad una regla global, categórica, del español hablado en la ciudad de La Habana. De ser categórica debería de cumplirse en todos los casos, mas la evidencia obtenida en este estudio no parece apoyar tal suposición, al menos en lo que concierne a las vocales medias. Los resultados de la medición de tiempo y la carta fonética de la Figura 3 permiten observar dónde está el problema que no capta ni la regla global de elisión de /s/ propuesta por Hammond ni la representación de Núñez Cedeño. Dicho problema es un caso de variación fonética en el que la aspiración de /s/ no es concomitante con el alargamiento de la vocal precedente ni con la apertura de dicho segmento. Por otro lado, lo que tenemos en el nivel prosódico es que la mora correspondiente a la /s/ aspirada, al no alargarse la vocal precedente, simplemente se pierde. 


\title{
Conclusiones
}

La evidencia acústica y estadística confirma que el alargamiento y la apertura de las vocales medias /e/ y /o/ del español hablado en la ciudad de La Habana son fenómenos sujetos a una amplia variación fonética. El alargamiento parece ser un epifenómeno, y la apertura un proceso no sistemático en el mejor de los casos, cuya manifestación no llega a ser estadísticamente significativa, contrario a lo que se esperaría dada la suposición común de que la aspiración y elisión de /s/ conlleva una apertura de la vocal precedente ${ }^{25}$. Lo observado fue que dicha apertura se dio, en el caso de /e/, cuando no estaba en contexto $\mathrm{de} / \mathrm{s} /$ aspirable, y en el caso de /o/, sólo en sílaba átona frente a /s/ aspirable. De ahí que no sea sencillo establecer una serie de realizaciones alofónicas "modelo" (por ejemplo, $[\mathrm{e}],[\varepsilon][\mathrm{o}]$, [॰], etc.), dada la variación fonética que mostraron los segmentos /e/ y /o/ en los contextos estudiados.

Por último, es necesario señalar que este acercamiento con evidencia acústica y estadística a la variación de las vocales medias del habanero nos indicó pistas más objetivas sobre la hipótesis de peso silábico respecto del vocalismo en esta variante del español, y demostró que el alargamiento vocálico fonético no necesariamente puede ser predicho a partir de una teoría basada en un sistema de reglas y/o representaciones. Objetivamente, no podemos rechazar por completo la ocurrencia del alargamiento en esta variante del español; sin embargo, el hecho de que no hayamos observado ni un proceso de alargamiento vocálico compensatorio, ni un proceso de apertura vocálica estadísticamente significativos, nos indica que los comportamientos fónicos en los procesos de variación se explican mejor si tomamos en cuenta distintos aspectos de la realidad fónica del sistema lingüístico, y no solamente las formas fonéticas de la melodía segmental.

\author{
Édgar A. MAdrid Servín \\ Elizabeth Santana Cepero \\ Universidad Autónoma Metropolitana-Iztapalapa
}

25 Nuestros resultados coinciden, en general, con las impresiones de Humberto López Morales (El español del Caribe, Mapfre, Madrid, 1992), quien manifiesta no estar de acuerdo ni con la existencia del desdoblamiento vocálico, ni con la del alargamiento en el español de Cuba. 


\section{APÉNDICE}

\section{ORACIONES GRABADAS QUE CONTIENEN LOS SEGMENTOS ANALIZADOS}

(1) El hombre pesca sardinas y pargos.

(2) Todos los días pesca con sus redes.

(3) Se dedica a la pesca de sardinas y pargos.

(4) Mi hijo peca de precavido muchas veces.

(5) También las gentes de pelos rojos y amarillos tienen pecas.

(6) Hay hombres que tienen patillas canosas y largas.

(7) Cuando tengo insomnio tomo pastillas para dormir.

(8) Me tomo dos aspirinas efervescentes grandes.

(9) El pescado que más me gusta es la cherna.

(10) Otro pescado que me gusta es el pargo.

(11) También pesco sardinas y otros pescados de mar.

(12) Es que en la costa también hay peces largos coloridos.

(13) Es que le decimos pez pargo en Cuba.

(14) Voy a buscarte por las tardes a la costa.

(15) Voy a la costa de Dos y Tercera.

(16) Después costeamos los boletos del cine.

(17) Al barco también se le dice buque de carga.

(18) Es que buque resulta una palabra muy fina.

(19) Es que hay acentos cubanos más finos que otros.

(20) Eva y Tere viven en un paraíso terrenal.

(21) Hay tres buques en los puertos isleños.

(22) En todos los puertos tenemos barcos grandes de eslora.

(23) En Cuba les llamamos fósforos a los cerillos de madera y a todos.

(24) Tengo treinta y un años de edad.

(25) Yo soy profesora de español y literatura.

(26) Doy clases en una escuela de nivel medio.

(27) También enseño a alumnos universitarios de nivel medio.

(28) Desarrollo diferentes actividades, todas a la vez.

(29) Tengo un puesto importante en esta escuela.

(30) Los baños de Artes y Letras tienen mucha peste.

(31) Yo no uso espejuelos nunca.

(32) En Cuba le dicen espejuelos a los lentes de armadura.

(33) Yo soy estudiante de Letras.

(34) En La Habana hay muchas escuelas primarias.

(35) En La Habana hay otros municipios más céntricos que éste.

(36) He ganado muchos conocimientos haciendo investigaciones.

(37) Actualmente estoy en un curso de profundización en el mismo centro.

(38) Mi hermano Alexis está en el año sabático.

(39) Cuéntame sobre tus estudios anteriores a la Maestría en Español.

(40) Me gusta más el francés que el inglés británico.

(41) Esos postes en la calle estorban la vista.

(42) Ellos son toscos y groseros latentes.

(43) Dos postes vio Óscar a lo lejos.

(44) Cuéntame de tus amores perdidos en el puerto.

(45) He hecho varios posgrados en literatura. 
(46) Las costumbres en La Habana se refieren a otros aspectos.

(47) Yo no padezco de osteoporosis.

(48) Estudié los costos comerciales.

(49) Nos enseñó todos los cuadros de fotos de familiares.

(50) Somos de treinta y tantos años de edad. 